\title{
Periarticular Bone Gain at Proximal Interphalangeal Joints and Changes in Bone Turnover Markers in Response to Tumor Necrosis Factor Inhibitors in Rheumatoid and Psoriatic Arthritis
}

\author{
Agnes Szentpetery, Malachi J. McKenna, Barbara F. Murray, Chin Teck Ng, Jennifer J. Brady, \\ Michelle Morrin, Bea Radovits, Douglas J. Veale, and Oliver FitzGerald
}

\begin{abstract}
Objective. Rheumatoid arthritis (RA) and psoriatic arthritis (PsA) are characterized by periarticular bone erosion; periarticular bone formation is a feature in PsA. The effect of anti-tumor necrosis factor- $\alpha$ (TNF- $\alpha)$ on periarticular bone remodeling is unclear in both diseases. Our aim was to assess the response of bone turnover markers (BTM) and hand bone mineral density (BMD) to anti-TNF over 3 years in RA and PsA.

Methods. We measured serum bone-specific alkaline phosphatase (bone ALP), procollagen type-I N-propeptide (PINP), intact osteocalcin, C-terminal cross-linking telopeptides (CTX-I), urinary N-terminal cross-linking telopeptide of type-I collagen (NTX-I), and free deoxypyridinoline crosslinks (fDPD) at baseline, 1, 12, and 36 months. BMD measurements (hands/spine/hip) were obtained at 3 timepoints.

Results. We recruited 62 patients (RA 35; PsA 27). BTM correlated significantly with hand BMD but not with central BMD. Low hand BMD was associated with RA and increased BTM. Following anti-TNF therapy, hip BMD declined while spine and hand BMD were unchanged. Periarticular BMD at proximal interphalangeal (PIP) joints increased while it decreased at metacarpophalangeal joints. Bone ALP increased steadily and was always higher in PsA. PINP and intact osteocalcin increased to a lesser extent, but resorption markers did not change.

Conclusion. At baseline, hand BMD was inversely associated with BTM. Bone formation rather than resorption markers better showed the bone response to anti-TNF. Despite a lack of effect on central BMD, the modest effect of anti-TNF on PIP BMD may provide evidence that BTM reflect specifically bone remodeling activity at periarticular sites of inflammation in RA and PsA. (First Release March 1 2013; J Rheumatol 2013;40:653-62; doi:10.3899/jrheum.120397)
\end{abstract}

\section{Key Indexing Terms: \\ PERIARTICULAR BONE REMODELING \\ HAND BONE MINERAL DENSITY \\ RHEUMATOID ARTHRITIS}

Rheumatoid arthritis (RA) and psoriatic arthritis (PsA) are chronic inflammatory diseases characterized by progressive destruction of the joints ${ }^{1,2,3}$. Inflammatory arthritis has the potential to affect bone biology at both local and systemic

From the Department of Rheumatology, the Metabolism Laboratory, and the DXA Unit, St. Vincent's University Hospital, Dublin, Ireland; and the Department of Medicine, University of Malaya, Kuala Lumpur, Malaysia. A. Szentpetery, MD, Department of Rheumatology, St. Vincent's University Hospital; M.J. McKenna, MD, FRCPI, Metabolism Laboratory and DXA Unit, St. Vincent's University Hospital; B.F. Murray, MSc, Metabolism Laboratory, St. Vincent's University Hospital; C.T. Ng, MD, Department of Rheumatology, St. Vincent's University Hospital, and Department of Medicine, University of Malaya; J.J. Brady, PhD; M. Morrin, BSc, Metabolism Laboratory, St. Vincent's University Hospital; B. Radovits, $M D$; D.J. Veale, MD, FRCP $(U K), F R C P I$, Professor of Rheumatology; O. FitzGerald, MD, FRCP(UK), FRCPI, Newman Clinical Research Professor, Department of Rheumatology, St. Vincent's University Hospital. Address correspondence to Prof. O. FitzGerald, Department of Rheumatology, St. Vincent's University Hospital, Elm Park, Dublin 4, Ireland.E-mail: oliver.fitzgerald@ucd.ie

Accepted for publication January 11, 2013.

\section{BONE TURNOVER MARKERS TUMOR NECROSIS FACTOR INHIBITOR PSORIATIC ARTHRITIS}

levels, given the links between inflammation and joint destruction $^{4,5,6}$. Local joint destruction has been investigated in depth in $\mathrm{RA}^{7}$. PsA shows profound differences in joint architecture, with a mixed pattern of concurrent erosions and new bone formation ${ }^{5}$. The mechanisms underlying periarticular bone loss include release of bone-resorbing cytokines from the inflamed synovium, increased vascularity, and immobility of affected joints ${ }^{8}$. Cytokine-mediated inflammatory processes rather than other factors lead to systemic bone loss in chronic arthritis in animal models ${ }^{9}$. Proinflammatory cytokines such as tumor necrosis factor- $\alpha$ (TNF- $\alpha$ ), interleukin 1 (IL-1), IL-6, IL-17, IL-18, IL-23, transforming growth factor- $\beta$, and tissue-degrading matrix metalloproteinases are believed to play essential roles in progressive joint damage ${ }^{6,10,11}$.

Erosion of periarticular bone is a central feature of both RA and PsA; the ability to measure its surrogates is important ${ }^{5,12}$. Several bone turnover markers (BTM) are useful in assessing joint damage in $\mathrm{RA}^{13,14,15,16,17,18,19,20,21}$. 
Yet there are fewer evaluations of bone and cartilage metabolism in PsA $22,23,24,25,26,27,28$. To date, no single BTM has been found to reflect joint destruction with sufficient accuracy to use in the clinic ${ }^{27}$. Information on the effect of anti-TNF on both localized and generalized osteoporosis in patients with RA is scarce $29,30,31$. Bone densitometry using dual-energy x-ray absorptiometry (DEXA) is known as an objective and precise method for monitoring bone loss, including that in the hands ${ }^{32,33,34}$. Measures of periarticular osteoporosis are more suitable for assessment of bone damage in RA because hand bone loss occurs earlier than generalized bone $\operatorname{loss}^{35}$. The rate of periarticular bone loss is higher than that in central sites and the precision of the DEXA is also superior at the hand compared with the hip and spine ${ }^{32}$. In RA, disease-related bone loss detected by DEXA occurs in the very early phase of the disease process, and hand bone loss is greater in patients with active disease $\mathrm{e}^{35,36,37}$. Despite periarticular osteoporosis also being a recognized clinical feature in PsA, only a few studies on systemic bone loss and bone turnover in PsA have been published $^{8,38,39}$.

The effect of anti-TNF on bone in RA and PsA has not been compared previously in a prospective study design. The primary aim of our study was to determine whether hand bone mineral density (BMD) increased following 3 years of anti-TNF treatment. The secondary aims were to study the relationship between BTM, hand BMD, and clinical markers of disease activity at baseline, to identify associations with hand BMD, to assess the response of BTM to 3 years of anti-TNF treatment, and to explore the relationship between changes in BTM and changes in disease activity after therapy.

\section{MATERIALS AND METHODS}

Patients. Our study was undertaken following approval by the St. Vincent's University Hospital Ethics and Medical Research Committee. Patients were recruited following informed consent from our Biologic Clinic, having been referred there for screening prior to the initiation of anti-TNF therapy. Inclusion criteria were diagnosis of RA or PsA according to American College of Rheumatology or ClASsification for Psoriatic ARthritis criteria (CASPAR), respectively, and age between 18 and 80 years ${ }^{40,41}$. All patients had ongoing active joint inflammation and had failed to respond adequately to at least 1 disease-modifying drug including maximum tolerated doses (up to $25 \mathrm{mg} /$ week) of methotrexate. Exclusion criteria included previous treatment with biologic agents 3 months prior to entering study, withdrawing from the biologic treatment during the study period, treatment with antiresorptive medications, parathyroid hormone or strontium ranelate 6 months prior to or during the study, diseases of bone metabolism, and pregnancy. The use of calcium and vitamin $\mathrm{D}$ supplements and a stable dose of steroids of $<10 \mathrm{mg} /$ day were permitted. Following screening, patients had their baseline clinical assessments performed and these were repeated after therapy at $3(n=57), 12(n=47)$, and 36 months $(n=51)$. Clinical assessments included a recording of the following: tender joint count (TJC), swollen joint count, patients' visual analog scale for global health, C-reactive protein (CRP), erythrocyte sedimentation rate (ESR), Health Assessment Questionnaire (HAQ), duration of early morning stiffness, and pain and fatigue scores by visual analog scale. A 28-joint Disease Activity Score (DAS28-CRP) was calculated and European League Against Rheumatism response criteria were applied ${ }^{42}$.
Biochemical measurements. Serum and urine samples were collected at baseline and after 1, 12, and 36 months of anti-TNF therapy, according to our laboratory protocol. Following an overnight fast, morning blood was collected in serum tubes containing a clot activator, and a second void urine sample was provided. These were measured as described ${ }^{43,44}$ : serum 25-hydroxy vitamin D [25(OH)D], parathyroid hormone (PTH), serum bone-specific alkaline phosphatase (bone ALP), procollagen type-I N-propeptide (PINP), intact osteocalcin, C-terminal cross-linking telopeptides (CTX-I), urinary N-terminal cross-linking telopeptide of type-I collagen (NTX-I), and free deoxypyridinoline crosslinks (fDPD). NTX-I and fDPD were expressed as a ratio with urine creatinine concentration, which was measured by a kinetic Jaffe method. A substudy was performed using a urinary biomarker of cartilage degradation, CTX-II. It was measured by enzyme immunoassay (Immunodiagnostic Systems, Urinary Cartilaps CTX-II). Intraassay coefficients of variation (CV) were $5.6 \%$ and $4.9 \%$ at concentrations of 1.25 and $3.26 \mathrm{ng} / \mathrm{ml}$, respectively. Interassay $\mathrm{CV}$ were $10.8 \%$ and $7.7 \%$ at concentrations of 1.58 and $4.26 \mathrm{ng} / \mathrm{ml}$, respectively. The results were expressed as CTX-II/creatinine ratio. Patients' results were compared with 21 healthy women and 5 men (mean age 31 years).

BMD measurements. BMD measurements were recorded using a Hologic Discovery A Model with software version 12.6. BMD assessments were obtained at left total hip, lumbar spine, and hands at baseline and 12 and 36 months. T scores and Z scores were calculated at hip using the US National Health and Nutrition Examination Survey III database and at spine using a manufacturer's database. Periarticular BMD of hands was measured as described $^{45}$. In brief, global hand BMD included all hand bones distal from the wrist joint; 7 subregions of interest were selected for analysis. These included the carpus and the periarticular regions of the second, third, and fourth MCP and PIP joints. The periarticular subregions of interest included $10 \mathrm{~mm}$ of bone proximal and distal to the joint line excluding the joint space, incorporating both the proximal and the distal juxtaarticular components of the joints (Figure 1). The mean BMD of the 14 regions in total (termed hand $\mathrm{BMD}$ ) and the average $\mathrm{BMD}$ at the 2 periarticular regions, MCP and PIP, were calculated.

Statistical methods. At baseline, differences between means were tested by either independent $t$ test, $t$ test for unequal variances, or Mann-Whitney $U$ test, where appropriate. Differences between categorical variables were tested by chi-square test. Associations between variables were tested by either Pearson or Spearman correlations. To identify associated variables, BMD at hand was divided at the median into 2 equal groups. Independent variables were tested by univariate single logistic regression analysis. Those variables with $\mathrm{p}<0.25$ were entered into a multivariable logistic regression model. Variables with a Wald statistic $>0.25$ were removed from the model; the remainder were retained based on testing differences between models using the likelihood ratio test. Given that hand BMD is a continuous variable, a multiple linear regression analysis was performed with independent variables being selected based on correlation analysis $(\mathrm{p}<0.05)$.

To assess the response to anti-TNF therapy, a repeated measures ANOVA was performed using a split-plot design, which tested between-subject and within-subject factors. The between-subject variable was the disease category (RA or PsA) and the within-subject variables included either 3 or 4 levels of the repeated measure factor as follows: 3 timepoints for BMD at hand $(n=45)$, spine $(n=45)$, and hip $(n=44) ; 4$ timepoints for BTM $(n=48)$; and 4 timepoints for clinical and inflammatory variables. In addition to testing the main effects, the interaction between each within-subject variable and disease category was tested. If significant differences were noted in the main effects, then pairwise comparisons of within-subject variables were performed and significance testing was adjusted for multiple comparisons according to Bonferroni correction. Results with $\mathrm{p}<0.05$ were considered statistically significant. Statistical analysis was performed using SPSS for Windows, Version 18. 


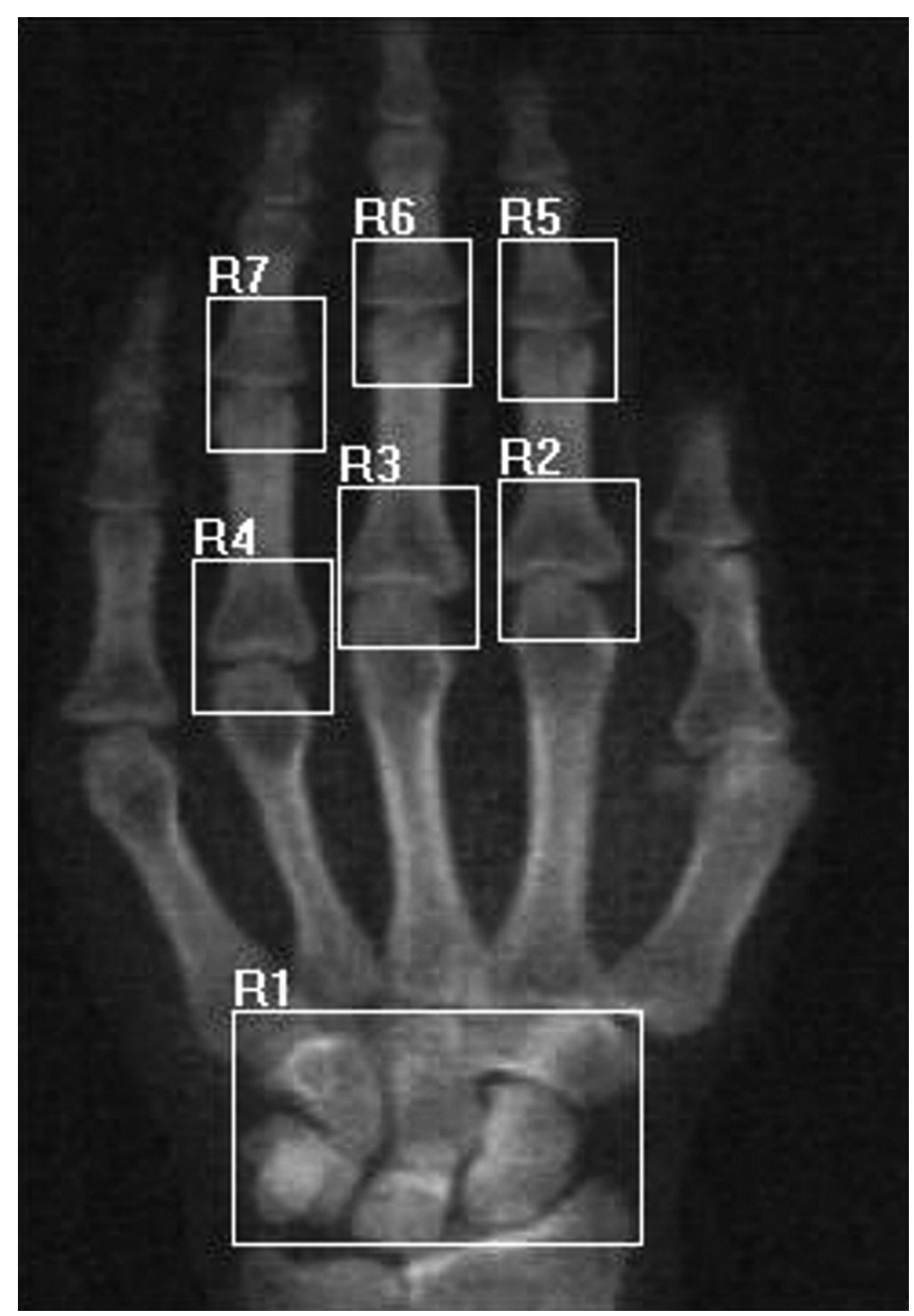

Figure 1. Dual-energy x-ray absorptiometry scan of left hand showing the 7 subregions of interest selected for analysis: carpus (R1) and the periarticular regions of the second (R2), third (R3), and fourth (R4) metacarpophalangeal joints and the second (R5), third (R6), and fourth (R7) proximal interphalangeal joints.

\section{RESULTS}

Baseline findings. We recruited 62 patients $(35 \mathrm{RA}, 27$ PsA). Patients with RA were older $(\mathrm{p}=0.022)$. ESR $(\mathrm{p}=$ $0.049)$ and CRP $(p=0.003)$ were significantly higher in RA compared to PsA (Table 1). DAS28-CRP reflected high disease activity in both groups at baseline. Clinical subtypes of patients with PsA were asymmetrical oligoarthritis $(\mathrm{n}=$ $15)$, symmetrical polyarthritis $(n=11)$, and predominant spondylitis $(n=1)^{46}$.

Serum ionized calcium was within the reference range in all subjects. Median serum 25(OH)D was $52.6 \mathrm{nmol} / \mathrm{l}$ (range $18.9-105.7 \mathrm{nmol} / 1) ; 16.4 \%$ of the patients were at risk of deficiency $(<30 \mathrm{nmol} / \mathrm{l})$ and $54.1 \%$ had sufficient levels $(\geq 50 \mathrm{nmol} / \mathrm{l})^{47}$. There was an inverse correlation between 25(OH)D and PTH $(r=-0.376, p=0.003)$. BTM did not correlate with any clinical disease activity scores. PINP correlated directly with both ESR $(r=0.257, \mathrm{p}<0.049)$ and CRP $(\mathrm{r}=0.284, \mathrm{p}<0.030)$. Urine fDPD correlated directly with both $\operatorname{ESR}(r=0.493, p<0.001)$ and CRP $(r=0.621, p<0.001)$. None of the other markers correlated with ESR or CRP.

Mean \pm SD urinary CTX-II/Cr in young adults was 258 $\pm 109 \mathrm{ng} / \mathrm{mmol}$; it was significantly higher than controls in a subgroup of 28 patients with RA $(681 \pm 517 \mathrm{ng} / \mathrm{mmol}$; $\mathrm{p}<$ $0.0001)$ and a subgroup of 23 patients with PsA (380 \pm 230 $\mathrm{ng} / \mathrm{mmol} ; \mathrm{p}=0.023)$. Levels were higher in RA than PsA $(\mathrm{p}=0.02)$. 
Table 1. Descriptive statistics at baseline. Results are presented as median (interquartile range), mean $\pm \mathrm{SD}$, or percentage.

\begin{tabular}{lccc}
\hline Characteristic & Total, $\mathrm{n}=62$ & $\mathrm{RA}, \mathrm{n}=35$ & PsA, $\mathrm{n}=27$ \\
\hline Demographic and clinical measures & & & \\
Age, yrs*** & $53(28-74)$ & $56(35-74)$ & $44(28-66)$ \\
Female:male, no. & $39: 23$ & $24: 11$ & $15: 12$ \\
Disease duration, yrs & $7(0.2-40)$ & $7(1-40)$ & $8(0.2-34)$ \\
Rheumatoid factor, $\mathrm{n}(\%)$ & $26(42)$ & $26(74)$ & $0(0)$ \\
Use of corticosteroids, $\mathrm{n}(\%)$ & $12(19.3)$ & $8(22.8)$ & $4(14.8)$ \\
BMI, kg/cm ${ }^{2}$ & $27.7 \pm 5.9$ & $26.2 \pm 6.4$ & $29.6 \pm 4.8$ \\
ESR, mm/h** & $25.8 \pm 23.9$ & $34.5 \pm 25.2$ & $15.1 \pm 17.3$ \\
CRP, mg/dl; normal <5** & $23.5 \pm 27.6$ & $32.1 \pm 32.9$ & $12.7 \pm 12.6$ \\
DAS28-CRP & $5.67(4.68-6.33)$ & $5.89(4.76-6.41)$ & $5.44(4.62-6.15)$ \\
Fatigue, 0-10 scale & $6(5-8)$ & $6(4.5-8.0)$ & $6(4.9-7.0)$ \\
GVAS, 0-100 mm VAS & $50(40-70)$ & $60(45-80)$ & $50(40-61)$ \\
HAQ, 0-3 scale & $1.13(0.63-1.63)$ & $1.38(0.63-1.75)$ & $1.06(0.59-1.50)$ \\
Pain, 0-10 scale $\dagger \dagger$ & $6.0(3.5-8.0)$ & $6(4.5-8.0)$ & $5.5(3.0-7.0)$ \\
TJC, 0-28 joints & $10(5-16)$ & $9(4.5-16.0)$ & $13(5-19)$ \\
SJC, 0-28 joints & $11.0(5.5-15.8)$ & $12(7.5-16)$ & $9(4-14)$ \\
Stiffness, min & $60(15-120)$ & $60(20-120)$ & $45(8.8-60)$ \\
Bone turnover markers & & & \\
Bone ALP, $\mu \mathrm{g} / \mathrm{l}^{\dagger}$ & $12.6 \pm 5.6$ & $10.8 \pm 3.6$ & $14.7 \pm 6.8$ \\
PINP, $\mu \mathrm{g} / \mathrm{l}$ & $49.2 \pm 22.9$ & $47.1 \pm 19.0$ & $51.8 \pm 27.2$ \\
OC[1-49], $\mu \mathrm{g} / 1$ & $12.0 \pm 6.5$ & $10.9 \pm 4.8$ & $13.3 \pm 8.1$ \\
CTX-I, $\mu \mathrm{g} / \mathrm{l}$ & $0.444 \pm 0.260$ & $0.444 \pm 0.234$ & $0.445 \pm 0.290$ \\
NTX-I, nmolBCE/mmol Cr & $47.5 \pm 31.9$ & $47.8 \pm 24.8$ & $47.1 \pm 39.7$ \\
fDPD, nmol/mmol Cr & $8.29 \pm 4.41$ & $9.05 \pm 4.79$ & $7.34 \pm 3.77$ \\
BMD measurements, g/cm ${ }^{2}$ & & & \\
Hand BMD & $0.350 \pm 0.052$ & $0.335 \pm 0.057$ & $0.360 \pm 0.047$ \\
PIP periarticular BMD & $0.285 \pm 0.051$ & $0.277 \pm 0.054$ & $0.294 \pm 0.043$ \\
MCP periarticular BMD & $0.305 \pm 0.51$ & $0.297 \pm 0.054$ & $0.316 \pm 0.040$ \\
Total hip BMD & $0.954 \pm 0.138$ & $0.936 \pm 0.141$ & $0.974 \pm 0.135$ \\
Lumbar spine BMD & $0.997 \pm 0.133$ & $0.989 \pm 0.137$ & $1.006 \pm 0.131$ \\
\hline
\end{tabular}

Differences between RA and PsA: $* \mathrm{p}<0.05 ; * * \mathrm{p}<0.01 ; * * * \mathrm{p}<0.001 .{ }^{\dagger}$ DAS28-CRP: 28 -joint Disease Activity Score using the C-reactive protein-based formula (www.das-score.nl). ${ }^{\text {\# }}$ Fatigue scale: $0=$ no fatigue to $10=$ fatigue as bad as it could be. ${ }^{\dagger \dagger}$ Pain scale: $0=$ no pain to $10=$ pain as bad as it could be. BMI: body mass index; ESR: erythrocyte sedimentation rate; GVAS: global visual analog scale $(0=$ worst imaginable health state to $100=$ best imaginable health state); HAQ: Health Assessment Questionnaire scale $(0=$ no difficulty to $3=$ unable to perform activity); TJC: tender joint count; SJC: swollen joint count; serum bone ALP: bone-specific alkaline phosphatase; serum PINP: procollagen type-I N-propeptide; serum OC[1-49]: intact osteocalcin; serum CTX-I: C-terminal cross-linking telopeptide of type-I collagen; urine NTX-I: N-terminal cross-linking telopeptide of type-I collagen; urine fDPD: free deoxypyridinoline crosslinks; BMD: bone mineral density; PIP: proximal interphalangeal joints; MCP: metacarpophalangeal joints.

According to World Health Organization criteria and based on BMD results expressed as T scores, osteopenia was evident in the spine in $22 \%$ and in the hip in $10 \%$, and osteoporosis was evident in the spine in $12 \%$ and in the hip in $2 \%$ at baseline (Table 1). There was good agreement between BMD measurements: spine versus hip $(r=0.659$, $\mathrm{p}<0.001)$, spine versus hand $(\mathrm{r}=0.515, \mathrm{p}<0.001)$, and hip versus hand $(r=0.657, p<0.001)$.

There were no significant correlations between either hip or spine BMD and any of the clinical measures of disease activity, or with BTM, even when analysis was repeated with BMD results expressed as T scores. In contrast, hand BMD correlated significantly and inversely with ESR $(r=$ $-0.217, \mathrm{p}=0.036)$, HAQ $(\mathrm{r}=-0.295, \mathrm{p}=0.023)$, bone ALP $(\mathrm{r}=-0.340, \mathrm{p}=0.007), \operatorname{PINP}(\mathrm{r}=-0.334, \mathrm{p}=0.009)$, CTX-I $(\mathrm{r}=-0.332, \mathrm{p}=0.008)$, NTX-I $(\mathrm{r}=-0.355, \mathrm{p}=0.008)$, and fDPD $(r=-0.351, p=0.005)$. Hand BMD also correlated with age $(r=-0.337, p=0.007)$ and weight $(r=0.470, p<$ 0.001). Thereafter analysis of variables was confined to the primary outcome measure, hand BMD.

These variables were associated with hand BMD according to univariate single logistic regression analysis ( $\mathrm{p}<0.05$; Table 2): disease category, sex, body weight, bone ALP, PINP, CTX-I, and NTX-I. After adjustment for disease category, odds ratios for association between BTM and hand BMD were tested; significant associations were noted for bone ALP, PINP, CTX-I, and NTX-I (supplementary data available from the author on request). Multivariable logistic regression analysis identified 3 associations with low hand BMD: presence of RA, lower body weight, and higher 
Table 2. Results of single variable and multivariable logistic regression analysis for the association between hand bone mineral density at baseline. OR refers to risk for hand bone mineral density below median.

\begin{tabular}{|c|c|c|c|c|}
\hline \multirow[b]{2}{*}{ Factor } & \multicolumn{2}{|c|}{ Single Variable Analysis } & \multicolumn{2}{|c|}{ Multivariable Analysis } \\
\hline & OR $(95 \% \mathrm{CI})$ & $\mathrm{p}$ & OR $(95 \% \mathrm{CI})$ & $\mathrm{p}$ \\
\hline Age, yrs & $0.96(0.91-1.01)$ & 0.089 & & \\
\hline Disease category* & $1.84(1.09-3.12)$ & 0.023 & $2.24(0.97-5.18)$ & 0.060 \\
\hline $\operatorname{Sex} * *$ & $3.66(1.22-11.0)$ & 0.021 & & \\
\hline Weight, kg & $1.05(1.01-1.10)$ & 0.016 & $1.06(1.01-1.12)$ & 0.042 \\
\hline $\mathrm{ESR}, \mathrm{mm} / \mathrm{h}$ & $0.98(0.95-1.00)$ & 0.060 & & \\
\hline CRP, mg/l & $0.99(0.97-1.01)$ & 0.281 & & \\
\hline DAS & $0.65(0.40-1.07)$ & 0.089 & & \\
\hline DAS28-CRP & $0.89(0.58-1.28)$ & 0.616 & & \\
\hline Fatigue, 0-10 scale & $0.96(0.75-1.22)$ & 0.732 & & \\
\hline GVAS, 0-100 mm & $0.92(0.72-1.17)$ & 0.492 & & \\
\hline HAQ, $0-3$ scale & $0.67(0.30-1.48)$ & 0.320 & & \\
\hline Pain, 0-10 scale & $1.16(0.92-1.45)$ & 0.203 & & \\
\hline TJC, $0-28$ joints & $0.99(0.93-1.05)$ & 0.659 & & \\
\hline SJC, 0-28 joints & $1.02(0.95-1.09)$ & 0.645 & & \\
\hline Stiffness, min & $1.00(0.99-1.01)$ & 0.500 & & \\
\hline Bone ALP, $\mu \mathrm{g} / 1$ & $0.87(0.77-0.99)$ & 0.031 & $0.76(0.620-0.94)$ & 0.010 \\
\hline PINP, $\mu \mathrm{g} / 1$ & $0.97(0.94-0.99)$ & 0.021 & & \\
\hline $\mathrm{OC}[1-49], \mu \mathrm{g} / 1$ & $0.92(0.83-1.02)$ & 0.099 & & \\
\hline CTX-I, $\mu \mathrm{g} / 1$ & $0.32(0.00-0.48)$ & 0.013 & & \\
\hline NTX-I, nmolBCE/mmol Cr & $0.97(0.95-0.99)$ & 0.026 & & \\
\hline $\mathrm{fDPD}, \mathrm{nmol} / \mathrm{mmol} \mathrm{Cr}$ & $0.88(0.77-1.01)$ & 0.070 & & \\
\hline
\end{tabular}

\footnotetext{
* If disease category is psoriatic arthritis. ** If sex is male. ESR: erythrocyte sedimentation rate; CRP: C-reactive protein; DAS28-CRP: 28-joint Disease Activity Score using the CRP; GVAS: global visual analog scale $(0=$ worst imaginable health state to $100=$ best imaginable health state); HAQ: Health Assessment Questionnaire scale ( $0=$ no difficulty to $3=$ unable to perform activity); TJC: tender joint count; SJC: swollen joint count; serum bone ALP: bone-specific alkaline phosphatase; serum PINP: procollagen type-I N-propeptide; serum OC[1-49]: intact osteocalcin; serum CTX-I: C-terminal cross-linking telopeptide of type-I collagen; urine NTX-I: N-terminal cross-linking telopeptide of type-I collagen; urine fDPD: free deoxypyridinoline crosslinks;
}

serum bone ALP (Table 2). The final model increased association with hand BMD from the chosen cutoff of $50 \%$ up to $77 \%$. The stepwise multiple linear regression analysis for the associations with hand BMD was significant $\left(r^{2}=\right.$ $0.446, \mathrm{p}<0.001)$ with the model also including disease category, weight, and bone ALP.

Response to anti-TNF therapy. The choice of anti-TNF therapy was at the discretion of the treating physician, resulting in 32 patients receiving etanercept, 28 adalimumab, and 2 infliximab. Combination therapy was given to $66 \%$ of the patients from baseline: methotrexate $(n=37)$, leflunomide $(n=2)$, hydroxychloroquine $(n=1)$, and sulfasalazine $(\mathrm{n}=1)$. Eight patients with RA $(22.8 \%)$ and 4 with PsA $(14.8 \%)$ received low-dose corticosteroids at various timepoints. We were unable to evaluate statistically the effect of concomitant corticosteroid use on BTM because of small sample size. In all, 26 women $(66.6 \%)$ were postmenopausal [19 (79.2\%) of those with RA and 7 (46.6\%) of those with PsA]. None of the patients used menopausal hormone therapy.

At 36 months, 51 patients completed the study (4 withdrew, 2 died, 5 left for other reasons); although varying numbers had complete datasets at all the timepoints for analysis by split-plot ANOVA (Tables 3, 4, and 5). For all the clinical measures, highly significant improvements were noted; disease category had a main effect on ESR only ( $\mathrm{p}=$ 0.021), with ESR being lower in PsA than RA. There was only 1 interaction effect, which was between disease category and TJC $(p=0.023)$. The effect on both ESR and CRP was waning at 3 years (Table 3, Figure 2). For BTM, significant changes were noted for bone ALP ( $p<0.001)$ and to a lesser extent for both PINP (p = 0.040) and intact osteocalcin ( $p=0.039)$; disease category had an effect only on bone ALP ( $\mathrm{p}=0.015)$, with bone ALP being higher in PsA than in RA at all timepoints; and the only interaction was between disease category and CTX-I, whereby CTX-I drifted downward in RA but increased in PsA ( $\mathrm{p}=0.037$; Table 4, Figure 2). In RA, after 1 year of treatment, the CTX-II levels fell significantly to $560 \pm 428 \mathrm{ng} / \mathrm{mmol}$ ( $\mathrm{p}<$ $0.05)$. After 3 years of treatment, the levels fell further to $387 \pm 262 \mathrm{ng} / \mathrm{mmol}(\mathrm{p}<0.005)$. In PsA, after 1 and 3 years of treatment, the CTX-II levels were lower but not significantly different from baseline levels $(331 \pm 147 \mathrm{ng} / \mathrm{mmol}$ and $342 \pm 224 \mathrm{ng} / \mathrm{mmol}$, respectively).

There were significant correlations between change in urine fDPD and both change in $\operatorname{ESR}(\mathrm{r}=0.721, \mathrm{p}<0.001)$

Personal non-commercial use only. The Journal of Rheumatology Copyright (C) 2013. All rights reserved. 
Table 3. Response of inflammatory markers and clinical measures to anti-tumor necrosis factor- $\alpha$ therapy. Results are given as median (interquartile range).

\begin{tabular}{lccccc}
\hline Measure & $\mathrm{n}$ & Baseline & 3 Months & 1 Year & 3 Years \\
\hline ESR, $\mathrm{mm} / \mathrm{h}$ & 46 & $19(8-30)$ & $5(2-10)^{* *}$ & $7(2-15)^{* *}$ & $18(8-30)$ \\
CRP, $\mathrm{mg} / \mathrm{l}$ & 46 & $12(7-29)$ & $4(4-7)^{* *}$ & $4(3-5)^{* *}$ & $4(4-8)$ \\
DAS28-CRP & 42 & $5.8(4.7-6.4)$ & $3.4(3.0-4.3)^{* *}$ & $2.1(1.6-3.3)^{* *}$ & $3.2(2.4-4.0)^{* *}$ \\
Fatigue, 0-10 scale & 44 & $6.5(4.0-8.0)$ & $4.0(2.3-5.0)^{* *}$ & $3.5(1.0-7.8)^{* *}$ & $4.0(3.0-7.8)^{*}$ \\
GVAS, 0-100 mm & 45 & $5.0(4.0-8.0)$ & $2.0(1.0-3.0)$ & $1.0(1.0-3.5)$ & $3.4(1.6-5.4)$ \\
HAQ, 0-3 & 44 & $1.2(0.5-1.8)$ & $0.8(0.1-1.3)^{* *}$ & $0.4(0.0-0.9)^{* *}$ & $0.5(0.1-1.1)^{* *}$ \\
Pain, 0-10 & 45 & $6.0(4.3-8.0)$ & $3.0(1.0-4.0)^{* *}$ & $2.0(1.0-3.5)^{* *}$ & $3.0(2.0-5.5)^{* *}$ \\
TJC, 0-28 joints & 46 & $12(7-16)$ & $0(0-3)^{* *}$ & $2(0-5)^{* *}$ & $3(1-6)^{* *}$ \\
SJC, 0-28 joints & 46 & $11.5(8.0-15.3)$ & $2.0(0.0-4.0)^{* *}$ & $0.0(0.0-3.0)^{* *}$ & $0.0(0.0-1.0)^{* *}$ \\
Stiffness, min & 45 & $60(18-90)$ & $9(0-20)^{* *}$ & $0(0-15)^{* *}$ & $7(0-18)^{* *}$ \\
\hline
\end{tabular}

Repeated measures differences noted for all variables at $\mathrm{p}<0.001$; differences compared with baseline: $* \mathrm{p}<0.01 ; * * \mathrm{p}<0.001$; posthoc significance tests are adjusted for multiple comparisons. Differences between RA and PsA noted for ESR ( $\mathrm{p}=0.021)$ only. ERS: erythrocyte sedimentation rate; CRP: C-reactive protein; DAS28-CRP: 28-joint Disease Activity Score using the CRP; GVAS: global visual analog scale $(0=$ worst imaginable health state to $100=$ best imaginable health state); HAQ: Health Assessment Questionnaire scale (0 $=$ no difficulty to $3=$ unable to perform activity); TJC: tender joint count; SJC: swollen joint count; RA: rheumatoid arthritis; PsA: psoriatic arthritis.

Table 4. Response of bone turnover markers to anti-tumor necrosis factor- $\alpha$ therapy $(\mathrm{n}=48)$. Results are given as mean $\pm \mathrm{SD}$.

\begin{tabular}{lcccc}
\hline Marker & Baseline & 1 Month & 1 Year & 3 Years \\
\hline Bone ALP, $\mu \mathrm{g} / \mathrm{l}$ & $12.4 \pm 5.9$ & $13.1 \pm 6.5$ & $15.9 \pm 7.7 * *$ & $21.2 \pm 11.1^{* *}$ \\
PINP, $\mu \mathrm{g} / \mathrm{l}$ & $48.4 \pm 23.7$ & $52.4 \pm 28.0$ & $55.7 \pm 34.2$ & $53.9 \pm 23.5$ \\
OC[1-49], $\mu \mathrm{g} / \mathrm{l}$ & $12.2 \pm 7.1$ & $12.6 \pm 6.3$ & $14.8 \pm 10.6$ & $12.3 \pm 5.5$ \\
CTX-I, $\mu \mathrm{g} / \mathrm{l}$ & $0.443 \pm 0.263$ & $0.422 \pm 0.242$ & $0.425 \pm 0.281$ & $0.471 \pm 0.285$ \\
NTX-I, $\mathrm{nmolBCE} / \mathrm{mmol} \mathrm{Cr}$ & $46.9 \pm 35.0$ & $43.6 \pm 26.4$ & $47.6 \pm 30.9$ & $45.7 \pm 28.2$ \\
fDPD, $\mathrm{nmol} / \mathrm{mmol} \mathrm{Cr}$ & $8.17 \pm 4.37$ & $7.70 \pm 3.99$ & $7.63 \pm 3.91$ & $7.99 \pm 4.44$ \\
PINP/CTX-I ratio, $\mathrm{mcg} / \mathrm{mcg}$ & $127 \pm 47$ & $139 \pm 55$ & $147 \pm 57$ & $131 \pm 55$ \\
\hline
\end{tabular}

Repeated measures differences noted for bone ALP ( $p<0.001)$, PINP $(p=0.04)$, and for OC $[1-49]$ ( $p=0.039$ ). Differences compared with baseline: $* * \mathrm{p}<0.001$; posthoc significance tests are adjusted for multiple comparisons. Differences between RA and PsA noted for bone ALP as a main effect $(p=0.015)$ that was significant at all timepoints on posthoc testing. Bone ALP: bone-specific alkaline phosphatase; PINP: procollagen type-I N-propeptide; OC[1-49]: intact osteocalcin; CTX-I: C-terminal cross-linking telopeptide of type-I collagen; urine NTX-I: N-terminal cross-linking telopeptide of type-I collagen; fDPD: free deoxypyridinoline crosslinks; RA: rheumatoid arthritis; PsA: psoriatic arthritis.

Table 5. Bone mineral density (BMD; $\mathrm{g} / \mathrm{cm}^{2}$ ) results after anti-tumor necrosis factor- $\alpha$ therapy. Results are given as mean $\pm \mathrm{SD}$.

\begin{tabular}{llcll}
\hline Location & $\mathrm{n}$ & Baseline & 1 Year & 3 Years \\
\hline Hand & 45 & $0.356 \pm 0.045$ & $0.357 \pm 0.046$ & $0.360 \pm 0.056$ \\
PIP periarticular & 45 & $0.287 \pm 0.047$ & $0.296 \pm 0.048^{* *}$ & $0.305 \pm 0.056^{* *}$ \\
MCP periarticular & 45 & $0.305 \pm 0.042$ & $0.299 \pm 0.043^{*}$ & $0.300 \pm 0.043$ \\
Total hip & 44 & $0.949 \pm 0.132$ & $0.952 \pm 0.146$ & $0.935 \pm 0.139^{*}$ \\
Lumbar spine & 45 & $0.949 \pm 0.131$ & $0.952 \pm 0.146$ & $0.936 \pm 0.139$
\end{tabular}

Repeated measures differences noted for periarticular PIP $(p=0.002)$, periarticular MCP $(p=0.016)$, and total hip $(\mathrm{p}=0.014)$. Differences compared with baseline: $* \mathrm{p}<0.05, * * \mathrm{p}<0.01$. Posthoc significance tests are adusted for multiple comparisons. No differences were noted between rheumatoid arthritis and psoriatic arthritis. PIP: proximal interphalangeal joints; MCP: metacarpophalangeal joints. 

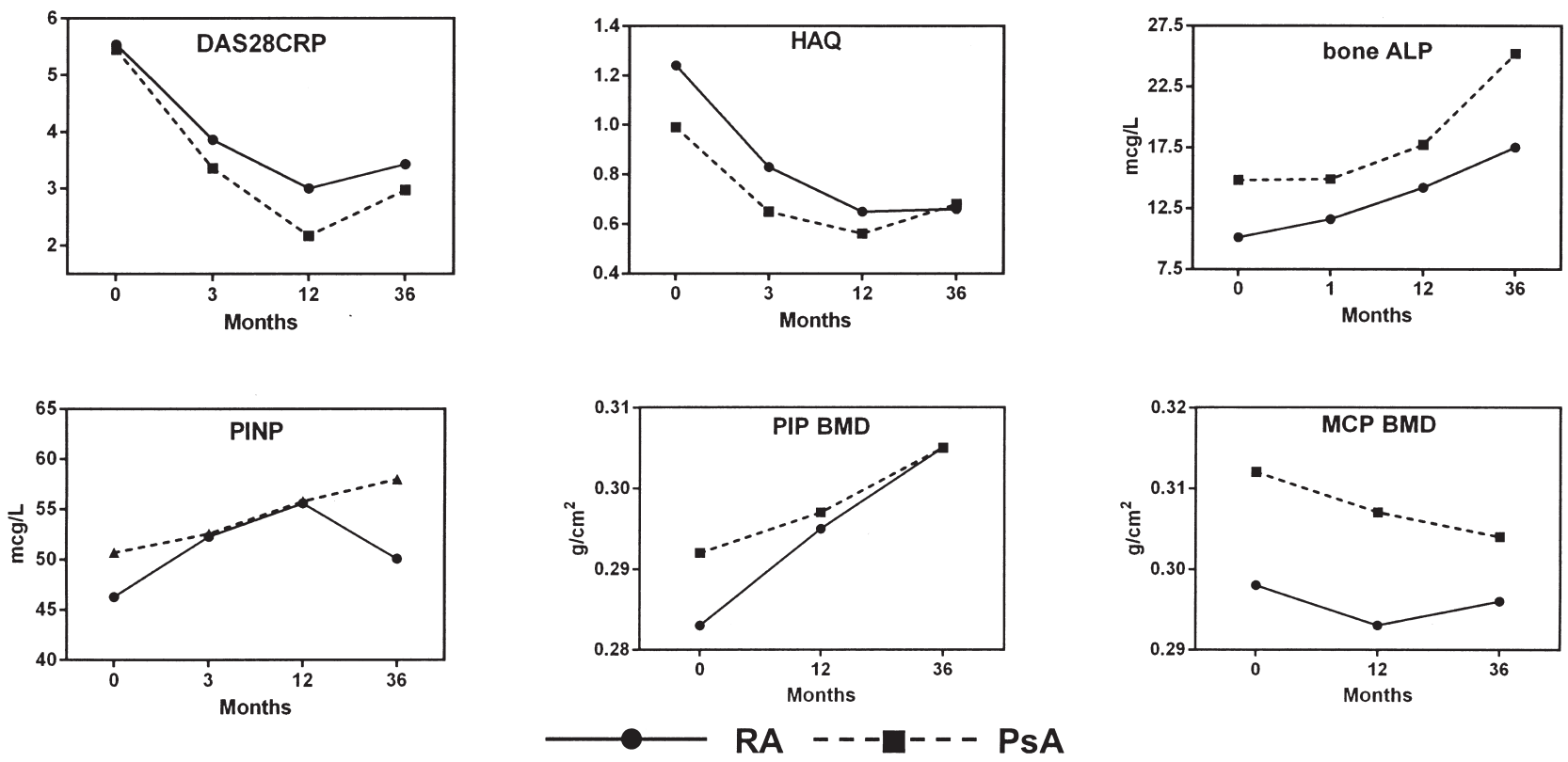

Figure 2. Response of selected variables to anti-tumor necrosis factor- $\alpha$ therapy, showing significant effects of treatment for all except metacarpophalangeal bone mineral density (MCP BMD), which shows an interaction effect. DAS28-CRP: 28-joint Disease Activity Score using C-reactive protein; HAQ: Health Assessment Questionnaire; bone ALP: bone-specific alkaline phosphatase; PINP: procollagen type-I N-propeptide; PIP: proximal interphalangeal.

and change in CRP $(\mathrm{r}=0.748, \mathrm{p}<0.001)$, but no similar changes were noted with the other BTM. We introduced an index that combined a formation and a resorption marker. To choose the best combination, we explored the correlation between changes in markers over the 3 years; the highest correlation was between PINP and CTX-I ( $\mathrm{r}=0.383, \mathrm{p}=$ $0.006)$. The index was expressed as a ratio $(\mu \mathrm{g} / \mu \mathrm{g})$ identical to a recently reported index ${ }^{48}$. There was no significant change in the index with anti-TNF therapy, but there was a definite trend toward positive remodeling balance that ameliorated at the time of the final measurement (Table 4).

For BMD, the within-subjects effects were seen for PIP, which increased $(\mathrm{p}=0.002)$; for hip, which declined $(\mathrm{p}=$ 0.014); and for MCP, which declined ( $\mathrm{p}=0.016$; Table 5, Figure 2). No effect of disease category was noted; there was an interaction between disease category and MCP BMD ( $p<0.001$ ), with BMD declining at the end of the first year for RA ( $p=0.073)$, whereas PsA showed a steady decline between baseline and third year $(\mathrm{p}=0.086$; Figure 2$)$.

\section{DISCUSSION}

At baseline, we identified associations between low hand BMD and the following variables: diagnosis of RA, lower body weight, higher serum bone ALP, higher PINP, higher CTX-I, and higher NTX-I. We found that baseline ESR, HAQ, and BTM correlated with hand BMD but not with hip or lumbar spine BMD. Thus, analysis at baseline suggested that joint inflammation is associated with remodeling activity and lower hand BMD. After 3 years of anti-TNF therapy, periarticular BMD increased significantly around
PIP joints but declined around MCP joints; hip BMD declined and spine BMD was unchanged. Bone ALP increased steadily over the 3 years and was consistently higher in PsA compared to RA; PINP and intact osteocalcin increased to a lesser degree. Resorption markers were unchanged; but change in urine fDPD was the only BTM to correlate with change in ESR. This suggests that anti-TNF therapy results in an increase in bone formation without a change in bone resorption; this would give a more favorable remodeling balance in periarticular bone.

We observed an inverse relationship between BTM and hand $\mathrm{BMD}$ at baseline on logistic regression analysis, suggesting that active joint inflammation alters periarticular bone remodeling with resultant bone loss because of negative remodeling balance. Following anti-TNF therapy, the steady increase in PIP periarticular BMD was contemporaneous with a steady increase in bone ALP and to a lesser extent in the 2 other formation markers - PINP and intact osteocalcin - implying a shift toward positive remodeling balance as a mechanism for periarticular bone gain. Bone ALP, being consistently higher in PsA compared to RA, reflects the known effect of PsA on bone formation, but the absence of an interaction effect between disease category and bone formation markers, as demonstrated by the split-plot ANOVA, suggests that the bone formation response occurs regardless of whether a patient has PsA or RA.

Regarding BTM in general, median levels at baseline of all markers except fDPD were below median levels in healthy Irish adults; and following intervention, median

Personal non-commercial use only. The Journal of Rheumatology Copyright (c) 2013. All rights reserved. 
values for bone ALP but not PINP or intact osteocalcin were above median levels in healthy Irish adults ${ }^{43}$. In our study cohort, both ESR and CRP correlated strongly with fDPD levels at baseline, indicating that fDPD is most likely an indicator of joint inflammation. Our results are in agreement with previous observations ${ }^{24,49}$. There were no correlations between ESR and CRP and the 2 other resorption markers, CTX-I and NTX-I. In addition, there were significant but weaker correlations between ESR and CRP with serum PINP, which is a collagen-based formation marker, but not with the non-collagen-based formation markers [serum intact osteocalcin and bone ALP]. Urinary CTX-II in a subgroup study has potential as a measure of cartilage degradation and as a response variable, at least in RA. The provider of this cartilage marker has recommended its use in RA and osteoarthritis only, and several studies have validated CTX-II measurements in these diseases ${ }^{16,50}$. CTX-II measurements have not previously been tested in PsA and our results indicate that it is not a useful marker for monitoring response to treatment in PsA.

Over 3 years of anti-TNF therapy, we demonstrated close correlations between changes in both ESR and CRP and change in urine fDPD, but no correlation with change in the other BTM. Similar associations between ESR and fDPD levels have been reported in patients with active RA treated with infliximab ${ }^{51}$. This indicates that urine fDPD is more likely a marker of joint inflammation than a marker of periarticular bone remodeling activity. Our data suggest that the effect of anti-TNF on bone is principally to suppress disease activity, which in turn alters bone remodeling at sites of joint inflammation.

From studies of bone histomorphometry using in vivo tetracycline, it is known that bone remodeling entails cycles of resorption coupled to formation ${ }^{52}$. RA is considered a purely erosive disease with little sign of repair of bone erosions, while PsA shows a mixed pattern of destruction and remodeling $5,7,53$. Bone remodeling is regulated by local and systemic factors that drive osteoblast and osteoclast differentiation and function ${ }^{7}$. Studies in RA have provided insights into the mechanism involved in the uncoupling of bone resorption and formation in this form of inflammatory $\operatorname{arthritis}^{54}$.

Data suggest that periarticular bone formation is actively suppressed by inflammation; thus, blockade of TNF is highly effective in retarding structural damage in $\mathrm{RA}^{5}$. It has been proposed that continued suppression of inflammation by anti-TNF agents may accelerate new bone formation in $\mathrm{PsA}^{55}$. Harrison, et al noted that in RA, but not in PsA, periarticular bone loss was strongly related to measures of joint inflammation ${ }^{36}$. We demonstrated that periarticular BMD around PIP joints improved in both diseases following 36 months of anti-TNF treatment, but not around MCP joints. This latter observation was rather unexpected. To our knowledge this is the first study comparing periarticular
BMD changes assessed in MCP and PIP joints in both RA and PsA as measured by DEXA following anti-TNF treatment. Finzel, et al have shown limited repair in bone erosions of the MCP joints in patients with RA treated with anti-TNF as measured by high-resolution computed tomography ${ }^{56}$. We did not find any marked differences in central BMD between the 2 diseases. After 3 years of anti-TNF therapy, hip BMD decreased and spine BMD remained stable. Anti-TNF treatment of mice transgenic for the human TNF gene leads to significant improvement in local bone resorption, but does not prevent generalized bone $\operatorname{loss}^{9,57}$. Future prospective studies might focus on RA and PsA patients with both early and established disease and compare axial, hand, and periarticular BMD because those results may have implications for prevention and treatment strategies of osteoporosis in inflammatory arthritis.

Our study design has some limitations. The study cohort consisted of patients with active disease; two-thirds of the patients received an anti-TNF agent in combination with a disease-modifying antirheumatic drug medication from baseline. Thus, we cannot fully confirm that the findings truly related to the effect of anti-TNF treatment. Yet data provided in our study appear to support this possibility. Our study was powered to detect a difference in hand BMD for a sample size of 60 , but only 45 subjects had repeated hand measures of BMD at the 3 timepoints. A larger sample size would be needed to validate our findings. We are proceeding with a further study.

Our study demonstrates that in a sample of patients with PsA or RA, there is a much closer association between markers of bone turnover with hand BMD than with central BMD at baseline. Following anti-TNF therapy over 3 years, periarticular PIP BMD increased but MCP BMD decreased. The increase in bone formation likely reflects an improvement in periarticular remodeling balance in both RA and PsA. The collagen-based marker of bone resorption, fDPD, seems to be altered by anti-TNF therapy as an antiinflammatory rather than as a bone remodeling effect. Despite a lack of effect on central BMD, the modest improvement in PIP BMD following anti-TNF therapy may indicate that BTM reflect specifically bone remodeling activity at sites of inflammation in RA and PsA.

\section{ACKNOWLEDGMENT}

The authors thank all the patients who participated in the study. The authors are also grateful to Phil Gallagher and Susan van der Kamp for their valuable contribution.

\section{REFERENCES}

1. Harris ED Jr. Rheumatoid arthritis. Pathophysiology and implications for therapy. N Engl J Med 1990;322:1277-89.

2. FitzGerald O, Kane D. Clinical, immunopathogenic, and therapeutic aspects of psoriatic arthritis. Curr Opin Rheumatol 1997;9:295-301.

3. Szekanecz Z, Koch AE. Update on synovitis. Curr Rheumatol Rep 2001;3:53-63. 
4. Murphy E, Roux-Lombard P, Rooney T, Fitzgerald O, Dayer JM, Bresnihan B. Serum levels of tissue inhibitor of metalloproteinase-1 and periarticular bone loss in early rheumatoid arthritis. Clin Rheumatol 2009;28:285-91.

5. Schett G. Osteoimmunology in rheumatic diseases. Arthritis Res Ther 2009;11:210.

6. Takayanagi H. Osteoimmunology and the effects of the immune system on bone. Nat Rev Rheumatol 2009;5:667-76.

7. Vandooren B, Yeremenko N, Noordenbos T, Bras J, Tak PP, Baeten D. Mediators of structural remodeling in peripheral spondylarthritis. Arthritis Rheum 2009;60:3534-45.

8. Frediani B, Allegri A, Falsetti P, Storri L, Bisogno S, Baldi F, et al. Bone mineral density in patients with psoriatic arthritis. J Rheumatol 2001;28:138-43.

9. Schett G, Redlich K, Hayer S, Zwerina J, Bolon B, Dunstan C, et al. Osteoprotegerin protects against generalized bone loss in tumor necrosis factor-transgenic mice. Arthritis Rheum 2003;48:2042-51.

10. Connell L, McInnes IB. New cytokine targets in inflammatory rheumatic diseases. Best Pract Res Clin Rheumatol 2006;20:865-78.

11. Cawston TE, Wilson AJ. Understanding the role of tissue degrading enzymes and their inhibitors in development and disease. Best Pract Res Clin Rheumatol 2006;20:983-1002.

12. Smolen JS, Aletaha D, Grisar J, Redlich K, Steiner G, Wagner O. The need for prognosticators in rheumatoid arthritis. Biological and clinical markers: where are we now? Arthritis Res Ther 2008;10:208.

13. Garnero P, Rousseau JC, Delmas PD. Molecular basis and clinical use of biochemical markers of bone, cartilage, and synovium in joint diseases. Arthritis Rheum 2000;43:953-68.

14. Garnero P, Thompson E, Woodworth T, Smolen JS. Rapid and sustained improvement in bone and cartilage turnover markers with the anti-interleukin-6 receptor inhibitor tocilizumab plus methotrexate in rheumatoid arthritis patients with an inadequate response to methotrexate: Results from a substudy of the multicenter double-blind, placebo-controlled trial of tocilizumab in inadequate responders to methotrexate alone. Arthritis Rheum 2010;62:33-43.

15. Chopin F, Garnero P, le Henanff A, Debiais F, Daragon A, Roux C, et al. Long-term effects of infliximab on bone and cartilage turnover markers in patients with rheumatoid arthritis. Ann Rheum Dis 2008;67:353-7.

16. Garnero P, Gineyts E, Christgau S, Finck B, Delmas PD. Association of baseline levels of urinary glucosyl-galactosyl-pyridinoline and type II collagen C-telopeptide with progression of joint destruction in patients with early rheumatoid arthritis. Arthritis Rheum 2002;46:21-30

17. Wislowska M, Jakubicz D, Stepień K, Cicha M. Serum concentrations of formation (PINP) and resorption (Ctx) bone turnover markers in rheumatoid arthritis. Rheumatol Int 2009;29:1403-9.

18. Kaufmann J, Mueller A, Voigt A, Carl HD, Gursche A, Zacher J, et al. Hydroxypyridinium collagen crosslinks in serum, urine, synovial fluid and synovial tissue in patients with rheumatoid arthritis compared with osteoarthritis. Rheumatology 2003;42:314-20.

19. Garnero P, Landewe R, Boers M, Verhoeven A, Van Der Linden S, Christgau $S$, et al. Association of baseline levels of markers of bone and cartilage degradation with long-term progression of joint damage in patients with early rheumatoid arthritis: The COBRA study. Arthritis Rheum 2002;46:2847-56.

20. Arends S, Spoorenberg A, Bruyn GA, Houtman PM, Leijsma MK, Kallenberg CG, et al. The relation between bone mineral density, bone turnover markers, and vitamin D status in ankylosing spondylitis patients with active disease: A cross-sectional analysis. Osteoporos Int 2011;22:1431-9.

21. Freeston JE, Garnero P, Wakefield RJ, Hensor EM, Conaghan PG,
Emery P. Urinary type II collagen C-terminal peptide is associated with synovitis and predicts structural bone loss in very early inflammatory arthritis. Ann Rheum Dis 2011;70:331-3.

22. Franck H, Ittel T. Serum osteocalcin levels in patients with psoriatic arthritis: An extended report. Rheumatol Int 2000;19:161-4.

23. Magarò M, Altomonte L, Mirone L, Zoli A, Tricerri A. Serum osteocalcin as an index of bone turnover in active rheumatoid arthritis and in active psoriatic arthritis. Clin Rheumatol 1989;8:494-8

24. Hein G, Schmidt F, Barta U, Müller A. Is there a psoriatic osteopathy? - The activity of bone resorption in psoriatics is related to inflammatory joint process. Eur J Med Res 1999; 4:187-92.

25. Mânsson B, Gülfe A, Geborek P, Heinegård D, Saxne T. Release of cartilage and bone macromolecules into synovial fluid: Differences between psoriatic arthritis and rheumatoid arthritis. Ann Rheum Dis 2001;60:27-31.

26. Fraser A, Fearon U, Billinghurst RC, Ionescu M, Reece R, Barwick $\mathrm{T}$, et al. Turnover of type II collagen and aggrecan in cartilage matrix at the onset of inflammatory arthritis in humans: Relationship to mediators of systemic and local inflammation. Arthritis Rheum 2003;48:3085-95.

27. Mullan RH, Matthews C, Bresnihan B, FitzGerald O, King L, Poole $\mathrm{AR}$, et al. Early changes in serum type II collagen biomarkers predict radiographic progression at one year in inflammatory arthritis patients after biologic therapy. Arthritis Rheum 2007;56:2919-28

28. van Kuijk AW, DeGroot J, Koeman RC, Sakkee N, Baeten DL, Gerlag DM, et al. Soluble biomarkers of cartilage and bone metabolism in early proof of concept trials in psoriatic arthritis: Effects of adalimumab versus placebo. PLoS One 2010;5:9.

29. Haugeberg G, Conaghan PG, Quinn M, Emery P. Bone loss in patients with active early rheumatoid arthritis: Infliximab and methotrexate compared with methotrexate treatment alone. Explorative analysis from a 12-month randomised, double-blind, placebo-controlled study. Ann Rheum Dis 2009;68:1898-901.

30. Wijbrandts CA, Klaasen R, Dijkgraaf MG, Gerlag DM, van Eck-Smit BL, Tak PP. Bone mineral density in rheumatoid arthritis patients 1 year after adalimumab therapy: Arrest of bone loss. Ann Rheum Dis 2009;68:373-6.

31. Eekman DA, Vis M, Bultink IE, Kuik DJ, Voskuyl AE, Dijkmans BA, et al. Stable bone mineral density in lumbar spine and hip in contrast to bone loss in the hands during long-term treatment with infliximab in patients with rheumatoid arthritis. Ann Rheum Dis 2011;70:389-90.

32. Haugeberg G, Green MJ, Quinn MA, Marzo-Ortega H, Proudman $\mathrm{S}$, Karim Z, et al. Hand bone loss in early undifferentiated arthritis: Evaluating bone mineral density loss before the development of rheumatoid arthritis. Ann Rheum Dis 2006;65:736-40.

33. Franck H, Gottwalt J. Associations with subregional BMD-measurements in patients with rheumatoid arthritis. Rheumatol Int 2008;29:47-51.

34. Bejarano V, Hensor E, Green M, Haugeberg G, Brown AK, Buch $\mathrm{MH}$, et al. Relationship between early bone mineral density changes and long-term function and radiographic progression in rheumatoid arthritis. Arthritis Care Res 2012;64:66-70.

35. Haugeberg G, Green MJ, Conaghan PG, Quinn M, Wakefield R, Proudman SM, et al. Hand bone densitometry: A more sensitive standard for the assessment of early bone damage in rheumatoid arthritis. Ann Rheum Dis 2007;66:1513-7.

36. Harrison BJ, Hutchinson CE, Adams J, Bruce IN, Herrick AL. Assessing periarticular bone mineral density in patients with early psoriatic arthritis or rheumatoid arthritis. Ann Rheum Dis 2002;61:1007-11

37. Hoff M, Haugeberg G, Odegard S, Syversen S, Landewe R, van der

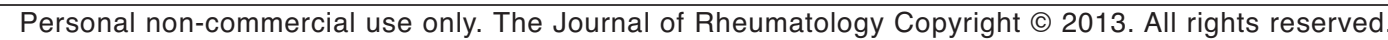


Heijde D, et al. Cortical hand bone loss after 1 year in early rheumatoid arthritis predicts radiographic hand joint damage at 5-year and 10-year follow-up. Ann Rheum Dis 2009;68:324-9.

38. Borman P, Babaoglu S, Gur G, Bingol S, Bodur H. Bone mineral density and bone turnover in patients with psoriatic arthritis. Clin Rheumatol 2008;27:443-7.

39. Dalbeth N, Pool B, Smith T, Callon KE, Lobo M, Taylor WJ, et al. Circulating mediators of bone remodeling in psoriatic arthritis: Implications for disordered osteoclastogenesis and bone erosion. Arthritis Res Ther 2010;12:R164.

40. Arnett FC, Edworthy SM, Bloch DA, McShane DJ, Fries JF, Cooper NS, et al. The American Rheumatism Association 1987 revised criteria for the classification of rheumatoid arthritis. Arthritis Rheum 1988;31:315-24.

41. Taylor W, Gladman D, Helliwell P, Marchesoni A, Mease P, Mielants H. Classification criteria for psoriatic arthritis: Development of new criteria from a large international study. Arthritis Rheum 2006;54:2665-73.

42. Fransen J, van Riel PL. The Disease Activity Score and the EULAR response criteria. Rheum Dis Clin North Am 2009;35:745-57, vii-viii.

43. Nanda KS, Ryan EJ, Murray BF, Brady JJ, McKenna MJ, Nolan N, et al. Effect of chronic hepatitis $\mathrm{C}$ virus infection on bone disease in postmenopausal women. Clin Gastroenterol Hepatol 2009; 7:894-9.

44. Waldron-Lynch F, Murray BF, Brady JJ, McKenna MJ, McGoldrick A, Warrington $\mathrm{G}$, et al. High bone turnover in Irish professional jockeys. Osteoporos Int 2010;21:521-5.

45. Murphy E, Bresnihan B, FitzGerald O. Measurement of periarticular bone mineral density in the hands of patients with early inflammatory arthritis using dual energy x-ray absorptiometry. Clin Rheumatol 2008;27:763-6.

46. Veale D, Rogers S, Fitzgerald O. Classification of clinical subsets in psoriatic arthritis. Br J Rheumatol 1994;33:133-8.
47. Institute of Medicine. Dietary reference intakes for calcium and vitamin D. Washington, DC: National Academies Press; 2011.

48. Josse AR, Atkinson SA, Tarnopolsky MA, Phillips SM. Diets higher in dairy foods and dietary protein support bone health during diet- and exercise-induced weight loss in overweight and obese premenopausal women. J Clin Endocrinol Metab 2012;97:251-60.

49. Grisar J, Bernecker PM, Aringer M, Redlich K, Sedlak M, Wolozcszuk W, et al. Ankylosing spondylitis, psoriatic arthritis, and reactive arthritis show increased bone resorption, but differ with regard to bone formation. J Rheumatol 2002;29:1430-6.

50. Garnero P, Charni N, Juillet F, Conrozier T, Vignon E. Increased urinary type II collagen helical and C telopeptide levels are independently associated with a rapidly destructive hip osteoarthritis. Ann Rheum Dis 2006;65:1639-44.

51. Torikai E, Kageyama Y, Takahashi M, Suzuki M, Ichikawa T, Nagafusa T, et al. The effect of infliximab on bone metabolism markers in patients with rheumatoid arthritis. Rheumatology 2006;45:761-4.

52. Recker RR. Bone biopsy and histomorphometry in clinical practice. Rheum Dis Clin North Am 1994;20:609-27.

53. Veale DJ, Ritchlin C, FitzGerald O. Immunopathology of psoriasis and psoriatic arthritis. Ann Rheum Dis 2005;64 Suppl 2:ii26-9.

54. Diarra D, Stolina M, Polzer K, Zwerina J, Ominsky MS, Dwyer D, et al. Dickkopf-1 is a master regulator of joint remodeling. Nat Med 2007;13:156-63.

55. Mensah KA, Schwarz EM, Ritchlin CT. Altered bone remodeling in psoriatic arthritis. Curr Rheumatol Rep 2008;10:311-7.

56. Finzel S, Rech J, Schmidt S, Engelke K, Englbrecht M, Stach C, et al. Repair of bone erosions in rheumatoid arthritis treated with tumour necrosis factor inhibitors is based on bone apposition at the base of the erosion. Ann Rheum Dis 2011;70:1587-93.

57. Redlich K, Hayer S, Maier A, Dunstan CR, Tohidast-Akrad M, Lang S, et al. Tumor necrosis factor alpha-mediated joint destruction is inhibited by targeting osteoclasts with osteoprotegerin. Arthritis Rheum 2002;46:785-92. 